\title{
Dust-penetrated morphology in the high-redshift universe: Clues from NGC 922
}

\author{
D. L. Block ${ }^{1}$, I. Puerari ${ }^{2}$, M. Takamiya ${ }^{3}$, R. Abraham ${ }^{4}$, A. Stockton ${ }^{5}$, I. Robson ${ }^{6}$, and W. Holland ${ }^{6}$ \\ 1 Dept. of Computational and Applied Mathematics, University of the Witwatersrand, Private Bag 3, \\ WITS 2050, South Africa \\ 2 Instituto Nacional de Astrofísica, Optica y Electrónica, Calle Luis Enrique Erro 1, 72840 Tonantzintla, Puebla, \\ México \\ 3 Gemini Observatory, 670 North Aohoku Place, Hilo, HI 96720, USA \\ 4 Department of Astronomy, University of Toronto, 60 St. George Str., Toronto, ON M5S 3H8, Canada \\ 5 Institute for Astronomy, University of Hawaii, 2680 Woodlawn Drive, Honolulu, HI 96822, USA \\ 6 Joint Astronomy Centre, Univ. Park, 660 North Aohoku Place, Hilo, HI 96720, USA
}

Received 16 November 2000 / Accepted 4 January 2001

\begin{abstract}
Results from the Hubble Deep Field (HDF) North and South show a large percentage of high-redshift galaxies whose appearance falls outside traditional classification systems. The nature of these objects is poorly understood, but sub-mm observations indicate that at least some of these systems are heavily obscured (Sanders 2000). This raises the intriguing possibility that a physically meaningful classification system for high-redshift galaxies might be more easily devised at rest-frame infrared wavelengths, rather than in the optical regime. Practical realization of this idea will become possible with the advent of the Next Generation Space Telescope (NGST). In order to explore the capability of NGST for undertaking such science, we present NASA-IRTF and SCUBA observations of NGC 922, a chaotic system in our local Universe which bears a striking resemblance to objects such as HDF 2-86 $(z=0.749)$ in the HDF North. If objects such as NGC 922 are common at highredshifts, then this galaxy may serve as a local morphological "Rosetta stone" bridging low and high-redshift populations. In this paper we demonstrate that quantitative measures of galactic structure are recoverable in the rest-frame infrared for NGC 922 seen at high redshifts using NGST, by simulating the appearance of this galaxy at redshifts $z=0.7$ and $z=1.2$ in rest-frame $K^{\prime}$. While this object cannot be classified within any optical Hubble bin, simulated NGST images at these redshifts can be readily classified using the dust penetrated $z \sim 0$ template of Block \& Puerari (1999) and Buta \& Block (2001). The near-infrared disk of NGC 922 is not peculiar at all; rather, it is remarkably regular, even presenting spiral arm modulation, a characteristic signature of several grand design galaxies. Our results suggest that the capability of efficiently exploring the rest-wavelength IR morphology of high- $z$ galaxies should probably be a key factor in deciding the final choice of instruments for the NGST.
\end{abstract}

Key words. galaxies: spiral - galaxies: structure - galaxies: kinematics and dynamics - galaxies: individual (NGC 922) - methods: numerical

\section{Introduction}

Considerable progress has recently been made in establishing the morphological mix of field galaxy populations as a function of redshift (e.g. Glazebrook et al. 1995; Driver et al. 1998; Abraham et al. 1996a). As a by-product of this work, a number of investigations probing the systematic changes in galaxy morphology as a function of rest-wavelength have been undertaken (Abraham et al. 1996b; Bouwens et al. 1998; Giavalisco et al. 1996). These studies have focused primarily on changes which occur as the rest-wavelength of observation moves from the optical

Send offprint requests to: D. L. Block, e-mail: block@cam.wits.ac.za regime into the near-ultraviolet (so-called "morphological K-corrections"). Remarkably little quantitative work has explored the systematic variations in morphology which occur as one moves from the optical in the other wavelength direction, namely into the near-IR. This is rather surprising, since the nearly constant mass-to-light ratio as a function of age for simple stellar populations suggests that near-IR morphology probes underlying stellar mass much more fairly than does optical morphology (Kauffmann \& Charlot 1998; Fig. 4 in Charlot 1998; Charlot 1996; Frogel et al. 1996).

The morphology of local galaxies can completely change once Population I disks are dust penetrated. One of many examples is NGC 5195, an optical irregular 


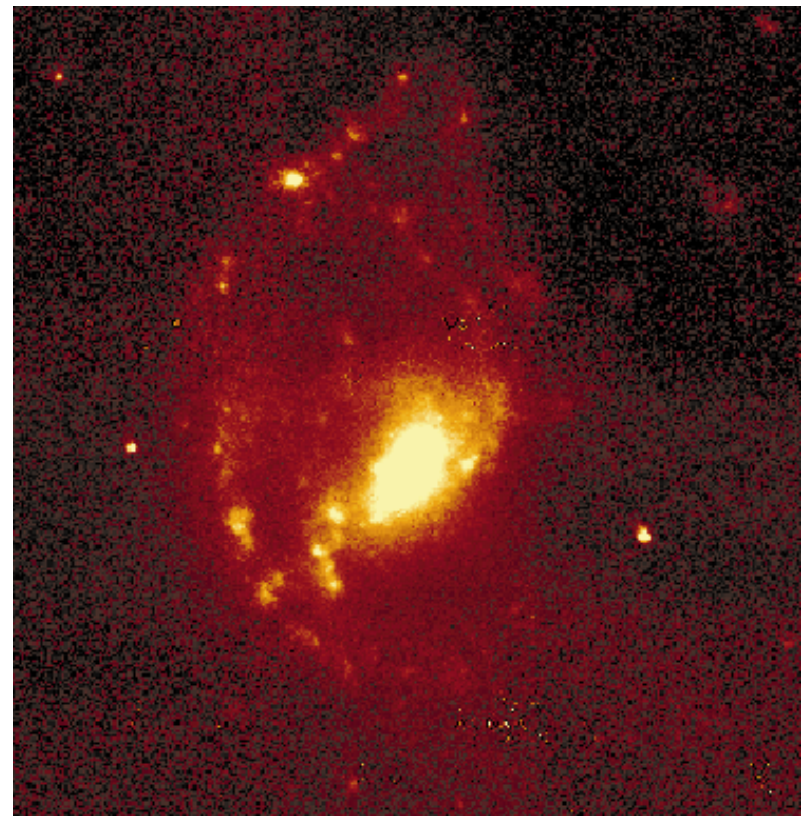

Fig. 1. A NASA IRTF near-infrared $\left(K^{\prime}\right)$ mosaic of NGC 922. An inner bisymmetric spiral, with wide open arms, lies within a prominent arc. The near-infrared disk of this optically chaotic specimen is remarkably regular: in fact, the inner spiral betrays the existence of arm modulation - a diagnostic feature of several grand design galaxies such as Messier 81

which presents an SBa morphology in the near-infrared. The extinction at $K^{\prime}(2.1 \mu \mathrm{m})$ is only $10 \%$ that in the $V$ $(0.55 \mu \mathrm{m})$ band. For local field galaxies, there is no correlation between dust penetrated classes and optical Hubble types; the Hubble tuning fork does not constrain the morphology of the old stellar Population II disks (Block \& Puerari 1999). Less is known at higher redshifts, since the evolved stellar disks of high- $z$ galaxies in the HDF have never been explored at restframe $K^{\prime}$ band. Images of galaxies with redshifts $z \sim 0.5-1$ or higher secured using the Hubble Space Telescope and NICMOS never penetrate the dusty, gaseous Population I mask. At $z>3$, even $H$ band $(1.6 \mu \mathrm{m})$ observed flux stems from emission shortward of $4000 \AA$. Early results from SCUBA suggest that a substantial proportion of high-redshift star-formation is occurring in heavily obscured galaxies (Sanders 2000; see also Fig. 5 in Hughes 1996).

Fortunately, upcoming advances in technology will soon allow the rest-frame IR morphology of galaxies to be probed at both low and high-redshifts. Near-infrared imaging surveys of local galaxies (such as the IR component of the VISTA survey) will be entirely comparable to those currently achievable at optical wavelengths. Similarly, the Next Generation Space Telescope (NGST) will allow studies of rest-wavelength IR morphology to be extended to high-redshifts. In this regard, the synergy between VISTA and NGST may turn out to be rather similar to that currently existing between ground-based optical facilities and HST.

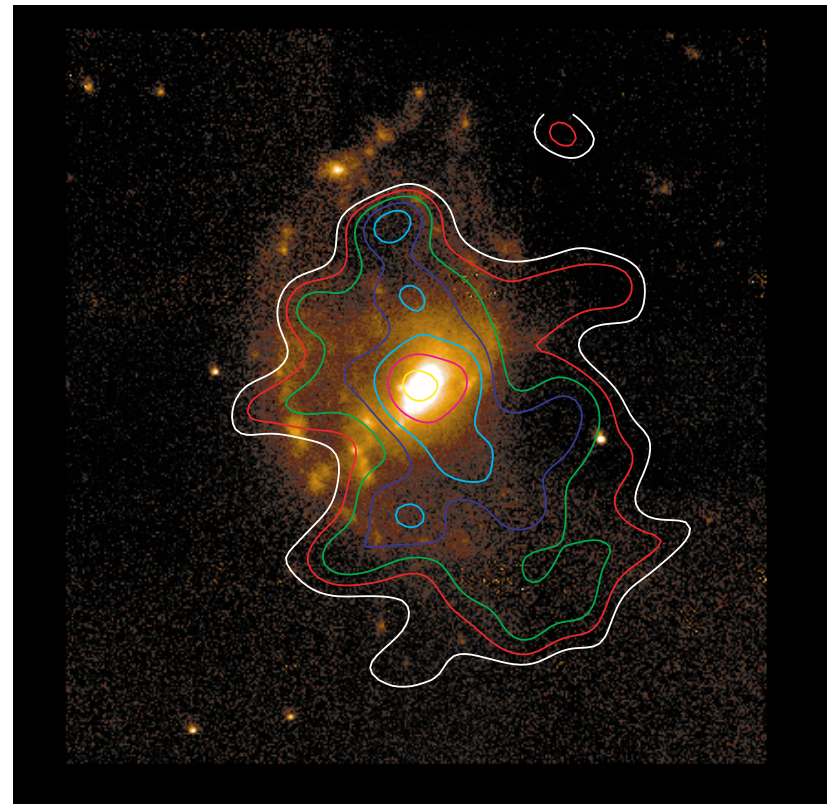

Fig. 2. $850 \mu \mathrm{m}$ SCUBA contours overlayed on the $K^{\prime}$ image. The contours start at $2 \sigma$ and increase in steps of $2 \sigma$. The peak lies at $27.5 \mathrm{mJy} /$ beam (beam $=14.4^{\prime \prime} 5 \mathrm{FWHM}$ ) and the noise level about $1.7 \mathrm{mJy} /$ beam $(\sim 14 \sigma)$. The integrated flux is $36 \mathrm{mJy}$, corresponding to a dust mass of $\sim 10^{7} M_{\odot}$

Motivated by this possibility, we have begun an investigation of the capability of NGST for extending local morphological studies to high redshifts. In this preliminary investigation, our strategy will be to focus on a single extreme case (NGC 922), which highlights the potential benefits of extending quantitative analyses of rest-wavelength near-IR morphology to high redshifts. This object is particularly well-suited for our purposes, since (in common with numerous high-redshift galaxies) "The morphology of NGC 922 is so peculiar as to be outside the classification system. It would be called a sport by nineteenthcentury animal breeders" (see Sandage \& Bedke 1994; Panel 313). This galaxy bears a striking optical resemblance to chaotic objects seen at high redshifts such as HDF 2-86 (van den Bergh 1998) at $z=0.749$ in the Hubble Deep Field (HDF), which van den Bergh et al. (1996) suggest might be a spiral in the process of being assembled. Both NGC 922 and HDF 2-86 contain a prominent arc-like feature in the one-half of the galaxy in which star formation is apparently proceeding at a vigorous rate (see Table 1 in Cohen 1976; Devereux 1989).

\section{Simulations of NGC 922 with NGST at Restframe $\mathrm{K}^{\prime}$}

$K^{\prime}$ images of NGC 922 were obtained at the NASA Infrared Telescope Facility with NSFCam, operating in the 0.3 arcsec mode, giving a field of $76^{\prime \prime}$. NGC 922 was placed successively in each quadrant of the detector in order to have good coverage of nearly uncontaminated blank sky, from which a median averaged sky flat could be obtained. Small dithers superposed on this basic 4-point 

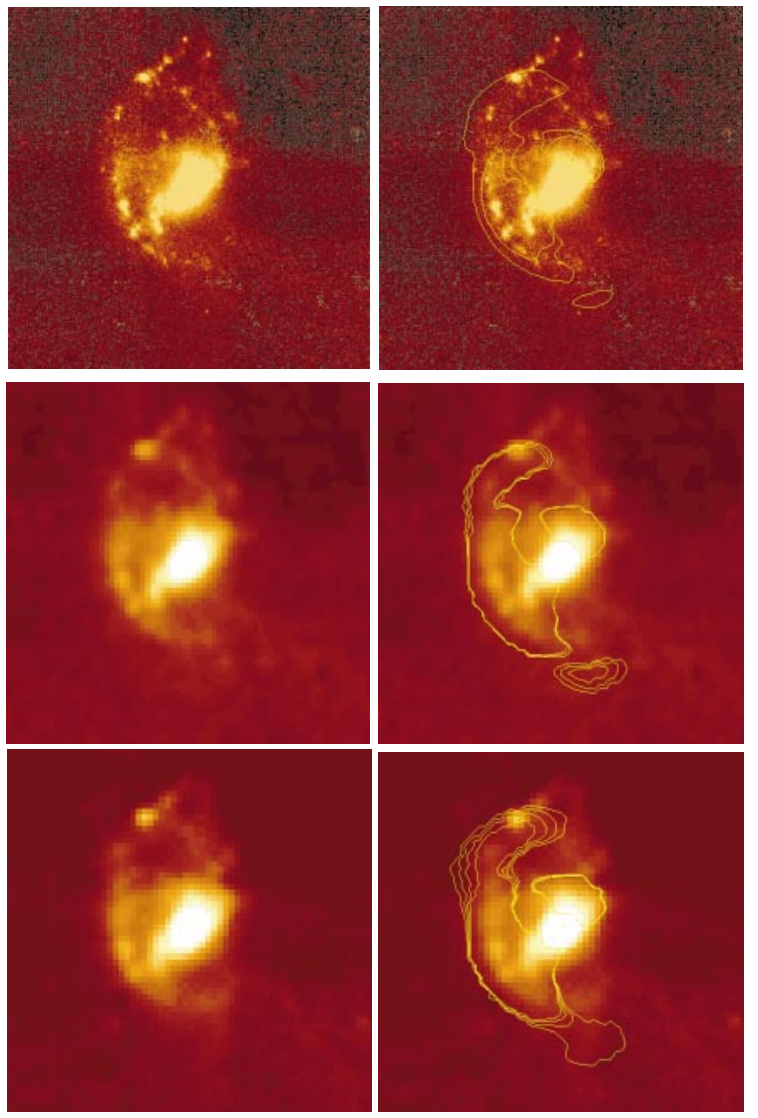

Fig. 3. NGC 922. Upper left: NGC 922, which optically falls outside the Hubble Classification scheme, imaged at $K^{\prime}$ with the NASA IRTF at Mauna Kea. Only two low-order modes ( $m=1$ and $m=2$ ) are required to quantify the structure in the stellar disk (see upper right, where the $m=1$ and $m=2$ contours, determined from the inverse Fourier transform, are overlayed on the groundbased $K^{\prime}$ image). Middle left: a simulated one hour image of NGC 922 with an $8 \mathrm{~m}$ NGST at a redshift $z=0.7$ ( $L$ band). Middle right: the $L$ band image, with $m=1$ and $m=2$ contours overlayed. Bottom right and left show the galaxy redshifted to $z=1.2$ ( $M$ band) with and without contours. These $M$-band postage stamp FITS images are only $3^{\prime \prime}$ on a side. For simulations illustrated here, a value of $q_{0}=0.1$ is assumed. Notice the power of the Fourier method to maintain the ubiquity of first and second modes in stellar disks (Block et al. 1994b), even for NGC 922

pattern ensured that stars in the field fell at different points of the detector each time. In all, 17 integrations of $1 \mathrm{~min}$ each were obtained. Our $K^{\prime}$ mosaic appears in Fig. 1.

The galaxy was also observed at $850 \mu \mathrm{m}$ using the Submillimetre Common-User Bolometer Array (SCUBA; see Holland et al. 1999) at the James Clerk Maxwell Telescope (JCMT) at Mauna Kea. The observing mode used was jiggle-mapping in which the secondary mirror of the telescope compensates for the instantaneous undersampled images by offsetting the array position to produce a Nyquist-sampled image. Some 300 separate "jigglemaps" (or integrations) were secured over a three night period, with a total on-source integration time of
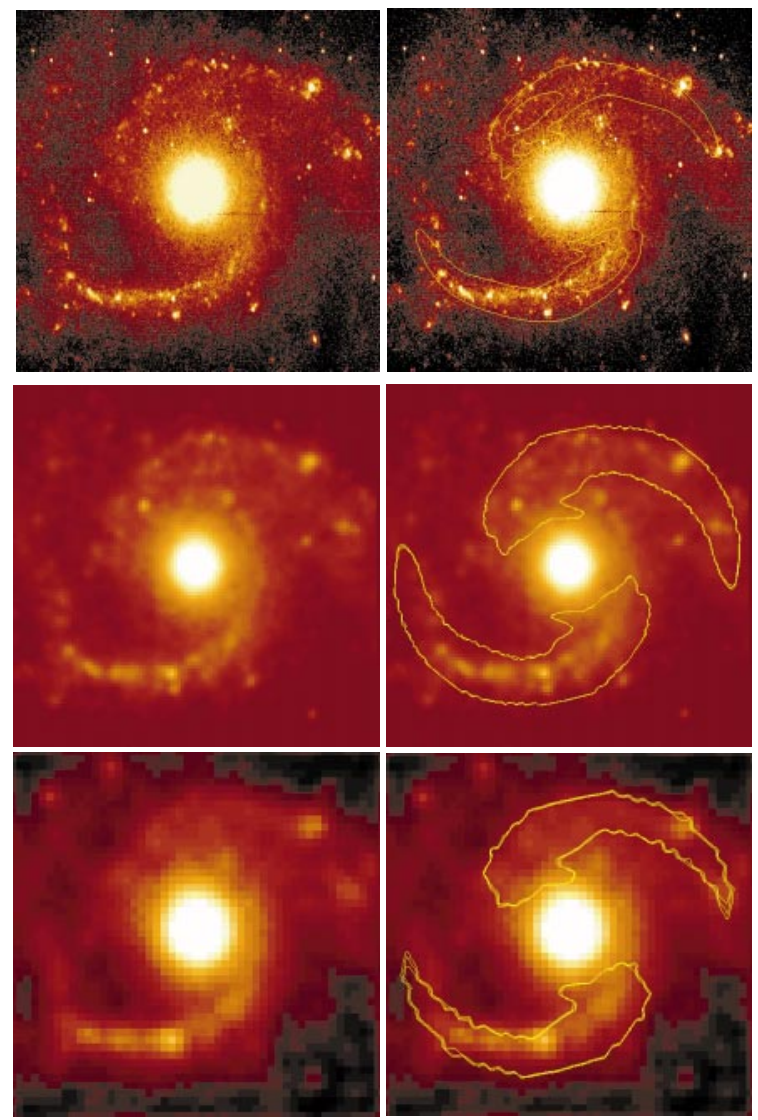

Fig. 4. NGC 2997. The original groundbased $K^{\prime}$ image is seen at upper left. Upper right: evensided $m=2$ contours determined from the inverse Fourier transform are overlayed on the groundbased $K^{\prime}$ image. Middle left: a simulated image of NGC 2997 with an $8 \mathrm{~m} \mathrm{NGST}$ at redshift $z=0.7$ ( $L$-band). Middle right: the $L$ band image, with contours (determined from the inverse Fourier transform) overlayed. Bottom right and left show the galaxy redshifted to $z=1.2$ ( $M$ band) with and without contours. The $M$-band images are only $\sim 2.5^{\prime \prime}$ on a side. Note the remarkable preservation of pitch angle with increasing redshift, and the power of the Fourier method to delineate spiral arms at restframe $K^{\prime}$ even at $z=1.2$

2.7 hours. The data was reduced using the SURF software package (Jenness \& Lightfoot 1998), correcting for noisy pixels, flatfield variations, atmospheric opacity, and short-time sky variations. The data were flux calibrated using beam-maps of Uranus and CRL618. The peak flux in the map is $25.0 \pm 1.7 \mathrm{mJy} /$ beam (following smoothing by a $7^{\prime \prime}$ Gaussian) and the integrated flux over a $100^{\prime \prime}$ diameter aperture, centered on the peak, is estimated to be $\sim 36$ mJy. Absolute calibration accuracy is estimated to be about $6 \%$.

Emission at $850 \mu \mathrm{m}$ is not confined to only one-half of the disk in which star-formation is occurring. The dust morphology is radically different to the near-infrared NASA-IRTF morphology (see Fig. 2); cold dust grains, seen in emission at $850 \mu \mathrm{m}$, are not restricted to the bright one-half of the almost "sliced-in-two" disk.

Assuming a mass absorption coefficient of 0.007 at $850 \mu \mathrm{m}$ and a grain temperature of $10-20 \mathrm{~K}$ (consistent 
with cold dust grain temperatures inferred using radiative transfer codes e.g. Block et al. 1994a), we compute an atomic and molecular hydrogen gas mass of $\sim 2-410^{9} M_{\odot}$. The dust mass for NGC 922 of $\sim 10^{7} M_{\odot}$, one order of magnitude greater than that of the "Evil Eye" Galaxy NGC 4826 (often cited as a prototype of a very dusty galaxy).

Our artificially redshifted images of the dusty NGC 922 are based on the preliminary description of the NGST facility given in Gillett \& Mountain (1998). For concreteness, we assumed an $8 \mathrm{~m}$ telescope. Our images assume an imager corresponding to the design of an $8192 \times 8192$ pixel format near-infrared camera operating on NGST from 0.6 to $5.3 \mu \mathrm{m}$ (which includes the $L$ and $M$ bands), as described in Bally \& Morse (1999). It is of course emphasized that at the present time the NGST telescope and camera designs have not been finalized, and the orbit placement of NGST not yet been decided. Indeed the present investigation of what can be achieved with the currently planned facility may provide some useful guidance to instrument designers.

In order to probe galaxies using NGST at redshifts 0.7 and 1.2 in their $K^{\prime}$ restframes, imaging detectors in the broadband $L(3.7 \mu \mathrm{m}$; bandpass $\delta L=0.65 \mu \mathrm{m})$ and $M(4.7 \mu \mathrm{m} ; \delta M=0.45 \mu \mathrm{m})$ regimes are required. At $M$, for example, the sky background at an excellent groundbased site such as Mauna Kea is 10 million times brighter than it will be for NGST (see Fig. 1 in Gillett \& Mountain 1998, where a sky background at Mauna Kea of $\sim 305$ Jansky $\operatorname{arcsec}^{-2}$ is adopted, but only $1.910^{-5}$ Jansky $\operatorname{arcsec}^{-2}$ for NGST).

We follow the simulation methodology of Takamiya (1999). The simulations recreate the images of NGC 922 when moved out to higher redshifts, always in a preselected restframe. In this paper, the images of NGC 922 are simulated at redshifts $z=0.7$ and $z=1.2$ in the dust penetrated $K^{\prime}$ rest-frame. Since the rest-frames are matched, no pixel $k$ corrections are applied (Takamiya 1999). If this was not the case, then $k$ corrections in simulations are necessary (e.g., Bouwens et al. 1998; Giavalisco et al. 1996). Furthermore, no spectral energy distribution templates are needed: these are only required for restframes which are not matched, such as when simulating HDF rest-frame UV images from local $z \sim 0$ optical ones (see for example, Abraham et al. 1996b).

We assume that the NGST sky background is about twice the minimum background observed by COBE (Hauser 1994), corresponding to a sky surface brightness at $L$ and $M$ of 19.55 and $17.31 \mathrm{mag}$ per square arcsecond, respectively. (From good near-infrared groundbased sites, the corresponding sky surface backgrounds at $L$ and $M$ are $\sim 5.99 \mathrm{mag} \operatorname{arcsec}^{-2}$ and $-0.24 \mathrm{mag} \operatorname{arcsec}^{-2}$, respectively.) We furthermore assume pixel sizes of 50 milliarcsec, a gain of 4 electrons per ADU (analog digital unit), a readout noise of 4 electrons and an output point spread function of Gaussian distribution, with a full width at half maximum $(F W H M)$ of 0 "' 12 . Assuming eight optical surfaces with 95\% transmission (these include the primary and secondary mirrors as well as lenses and filters in the detector) and a 40\% detector quantum efficiency, the system throughput is taken to be $26 \%$. An on-source integration time of one hour was adopted. Results from these simulations are shown in the left hand column of Fig. 3. A morphological decomposition based on these images is shown in the right hand column of this figure, and will be described in the next section. As a comparison, we ran a set of simulations on a galaxy which can readily be classified in the optical regime: NGC 2997. NGC 2997 shows a magnificent grand design structure in the optical regime; it is of Hubble type Sc and belongs to van den Bergh luminosity class I bin. The results of the NGC 2997 simulations are presented in Fig. 4.

\section{Morphological classification}

In the present paper we adopt the dust penetrated classification scheme for local spirals proposed by Block \& Puerari (1999) and Buta \& Block (2001). In the nearinfrared, the morphology of the older star dominated disk indicates a simple classification scheme (see Figs. 5 and 7): $\mathrm{H} m$ (where $m$ is the dominant Fourier harmonic), three pitch angle classes $\alpha, \beta$ and $\gamma$ and thirdly, a bar strength parameter. Bar strengths are derived from the gravitational potential or torque of the bar, and not from bar ellipticity. A bar which might be highly elongated (and therefore "strong" in optical images) may sometimes have a negligible bar strength, as inferred from its mass distribution and potential in near-infrared images (Buta \& Block 2001). We will show that the dust-penetrated tuning fork template for local galaxies shown in Fig. 7 readily accommodates NGC 922.

The 2-D Fast Fourier decomposition of NGC 922 images uses a program developed by I. Puerari (Schröder et al. 1994). Following Danver (1942) and Kalnajs (1975), logarithmic spirals of the form $r=r_{\mathrm{o}} \exp \left(-m \theta / p_{\max }\right)$ are employed in the decomposition.

The amplitude of each Fourier component is given by (Schröder et al. 1994)

$A(m, p)=\frac{\Sigma_{i=1}^{I} \Sigma_{j=1}^{J} I_{i j}(\ln r, \theta) \exp (-i(m \theta+p \ln r))}{\Sigma_{i=1}^{I} \Sigma_{j=1}^{J} I_{i j}(\ln r, \theta)}$

where $r$ and $\theta$ are polar coordinates, $I(\ln r, \theta)$ is the intensity at position $(\ln r, \theta), m$ represents the number of arms or modes, and $p$ is the variable associated with the pitch angle $P$, defined by $\tan P=-\frac{m}{p_{\max }}$.

After having deprojected the $K^{\prime}$ images and identifying the dominant modes, we calculate the inverse Fourier transform, as follows:

We define the variable $u \equiv \ln r$. Then

$S(u, \theta)=\sum_{m} S_{m}(u) \mathrm{e}^{i m \theta}$

where

$S_{m}(u)=\frac{D}{\mathrm{e}^{2 u} 4 \pi^{2}} \int_{-\infty}^{+\infty} G_{m}(p) A(p, m) \mathrm{e}^{i p u} \mathrm{~d} p$ 


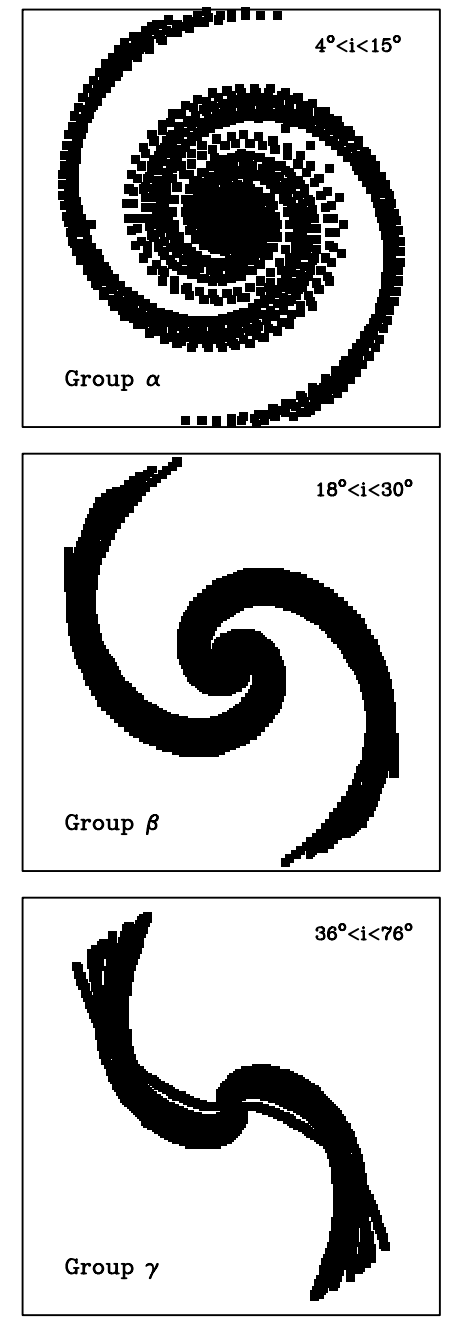

Fig. 5. Old disks may be grouped into three principal archetypes: the tightly wrapped $\alpha$ class, an intermediate $\beta$ class and an open $\gamma$ bin. These dust penetrated classes are related to the rate of shear in the stellar disk, as determined by $A / \Omega$, where $A$ is the first Oort constant and $\Omega$ is the circular frequency. Actual deprojected $K^{\prime}$ images of $\sim 50 \mathrm{H} 1$ and $\mathrm{H} 2$ galaxies have been superimposed to generate this template. Galaxies of class H1 (the first Fourier harmonic) present one dominant arm with a pitch angle belonging to one of the three classes $\alpha, \beta$ or $\gamma$. Galaxies of class H2 (the second Fourier harmonic) present two prominent spiral arms of pitch angle class $\alpha, \beta$ or $\gamma$

and

$D=\Sigma_{i=1}^{I} \Sigma_{j=1}^{J} I_{i j}(u, \theta)$

$G_{m}(p)$ is a high frequency filter used by Puerari \& Dottori (1992). For the spiral with $\tan P=-\frac{m}{p_{\max }^{m}}$ it has the form

$G_{m}(p)=\exp \left[-\frac{1}{2}\left(\frac{p-p_{\max }^{m}}{25}\right)^{2}\right]$

where $p_{\max }^{m}$ is the value of $p$ for which the amplitude of the Fourier coefficients for a given $m$ is maximum. This filter is also used to smooth the $A(p, m)$ spectra at the interval ends (see Puerari \& Dottori 1992).

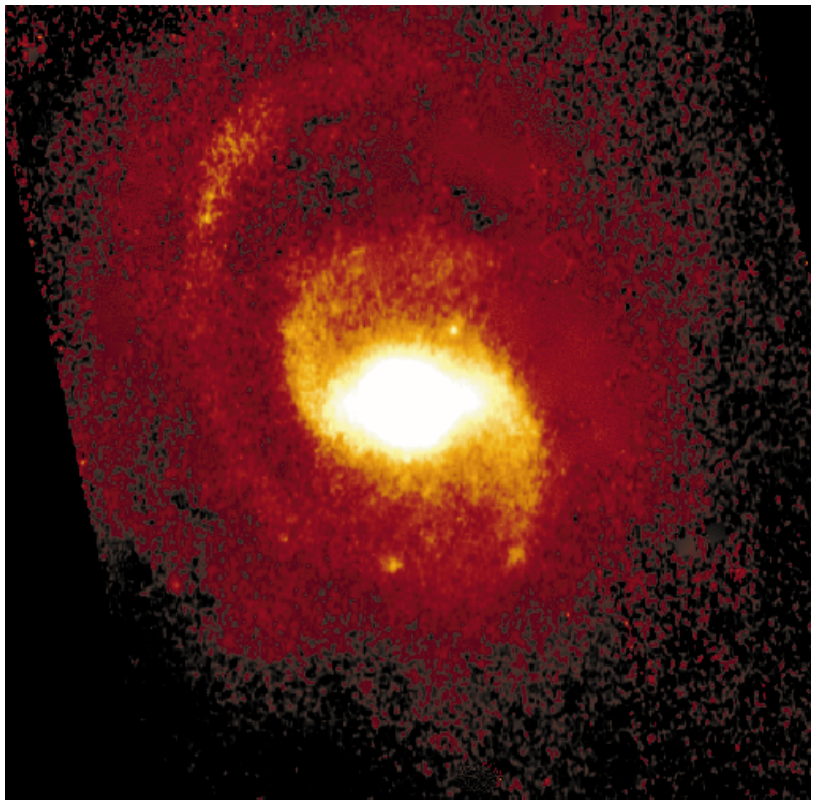

Fig. 6. In our local Universe, one of the most well studied lopsided spirals is NGC 1637, seen imaged here in the nearinfrared. The pitch angle of the lopsided arm is $\sim 35^{\circ}$; the arm belongs to the wide open $\gamma$ class. In NGC 922, the single arc as well as the inner bisymmetric spiral in Fig. 1 all belong to the $\gamma$ bin

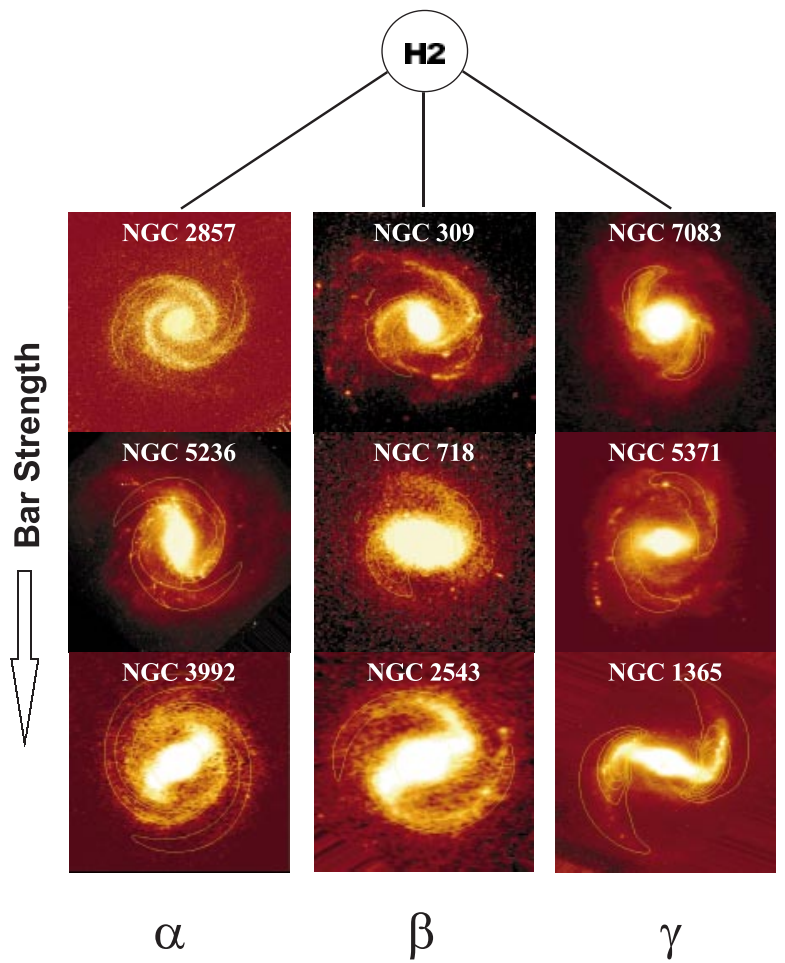

Fig. 7. In this dust penetrated $z \sim 0$ tuning fork template, galaxies are binned according to three quantitative criteria: $\mathrm{H} m$, where $m$ is the dominant Fourier harmonic (illustrated here are the two-armed $\mathrm{H} 2$ family); the pitch angle families $\alpha, \beta$ and $\gamma$ determined from the Fourier spectra, and finally the bar strength, derived from the gravitational potential (and not ellipticity) of the bar. Early type "b" galaxies NGC 3992, NGC 2543, NGC 7083, NGC 5371 and NGC 1365 are distributed within all three families $(\alpha, \beta$ and $\gamma)$. Adapted from Buta \& Block (2001) 

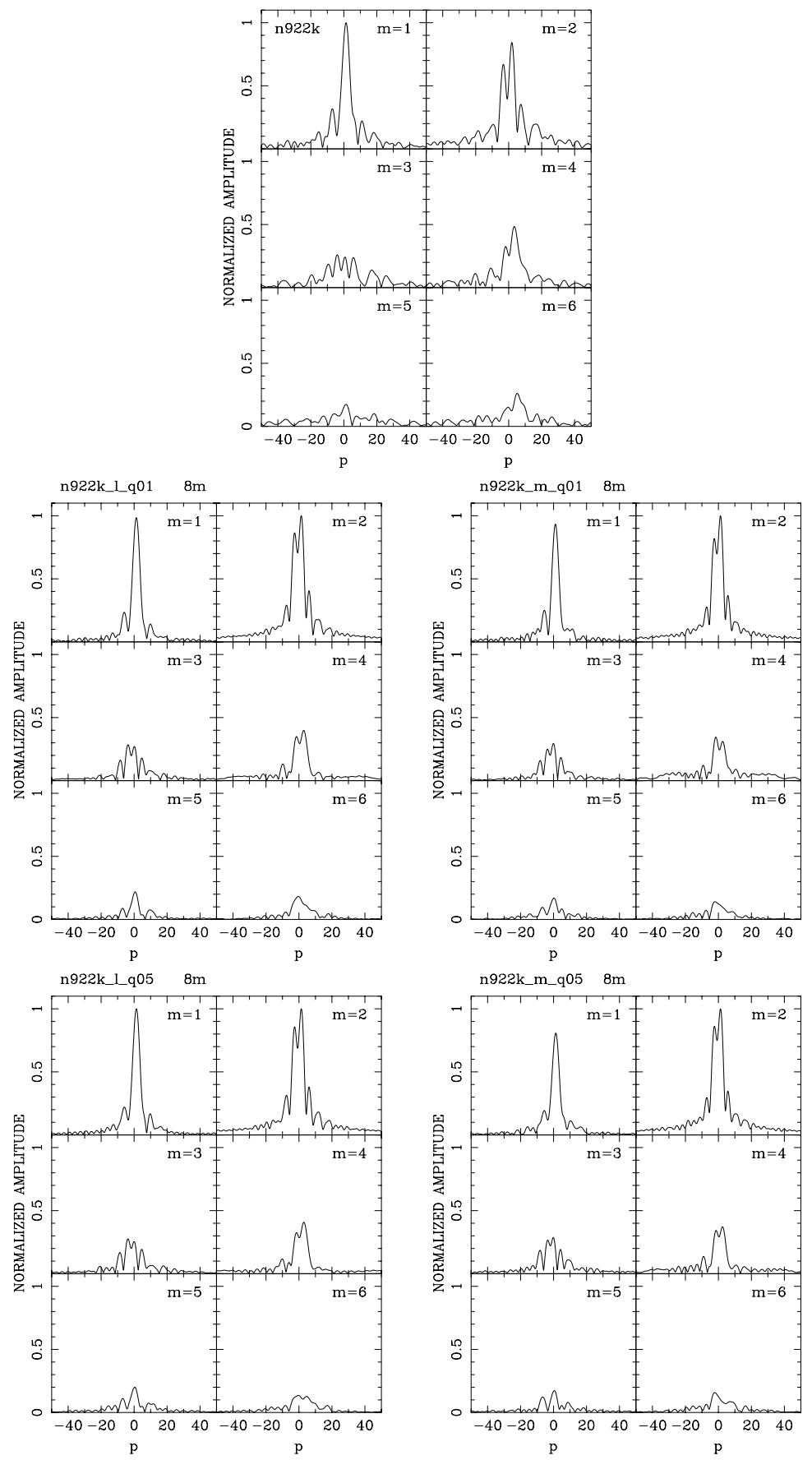

Fig. 8. The Fourier spectra of the $K^{\prime}$ image of NGC 922 at its unredshifted distance is shown at top. The middle row shows the Fourier spectra when NGC 922 is moved to redshifts $z=0.7$ ( $L$ band) and $z=1.2$ ( $M$ band), respectively. We assume that a class $8 \mathrm{~m}$ NGST has been used for securing the images, and that the on source integration time is one hour. We adopt a deceleration parameter of $q_{0}=0.1$. The bottom row shows the Fourier spectra for the same redshift values, but assuming a different cosmology with $q_{0}=0.5$. A remarkable similarity in the restframe $K^{\prime}$ images of NGC 922 is found, independent of redshift and of the deceleration parameters assumed. Note that the key signature for spiral arm modulation of the $m=2$ components $p>0$ and $p<0$ is beautifully retained into higher redshift space

NGC 922 is close to face-on; deep optical CCD images present an almost circular arc/disk. No deprojection corrections were therefore necessitated. Fourier spectra were determined for NGC 922 at $z=0.7$ and $z=1.2$ (see Fig. 8), and inverse Fourier transform contours were then overlayed on the simulated restframe $K^{\prime}$ mosaics, following the prescription above. The right hand columns of
Figs. 3 and 4 show the results of the inverse Fourier contour overlays on our one-hour NGST simulations.

The Fourier spectra of NGC 922 (see Fig. 8) shows a strong lopsided $m=1$ component, with a pitch angle corresponding to $39^{\circ}$ (i.e. class $\gamma$ in Fig. 5). The $m=1$ component is associated with the arc so clearly seen in our NASA-IRTF image. The $m=2$ component is almost as 

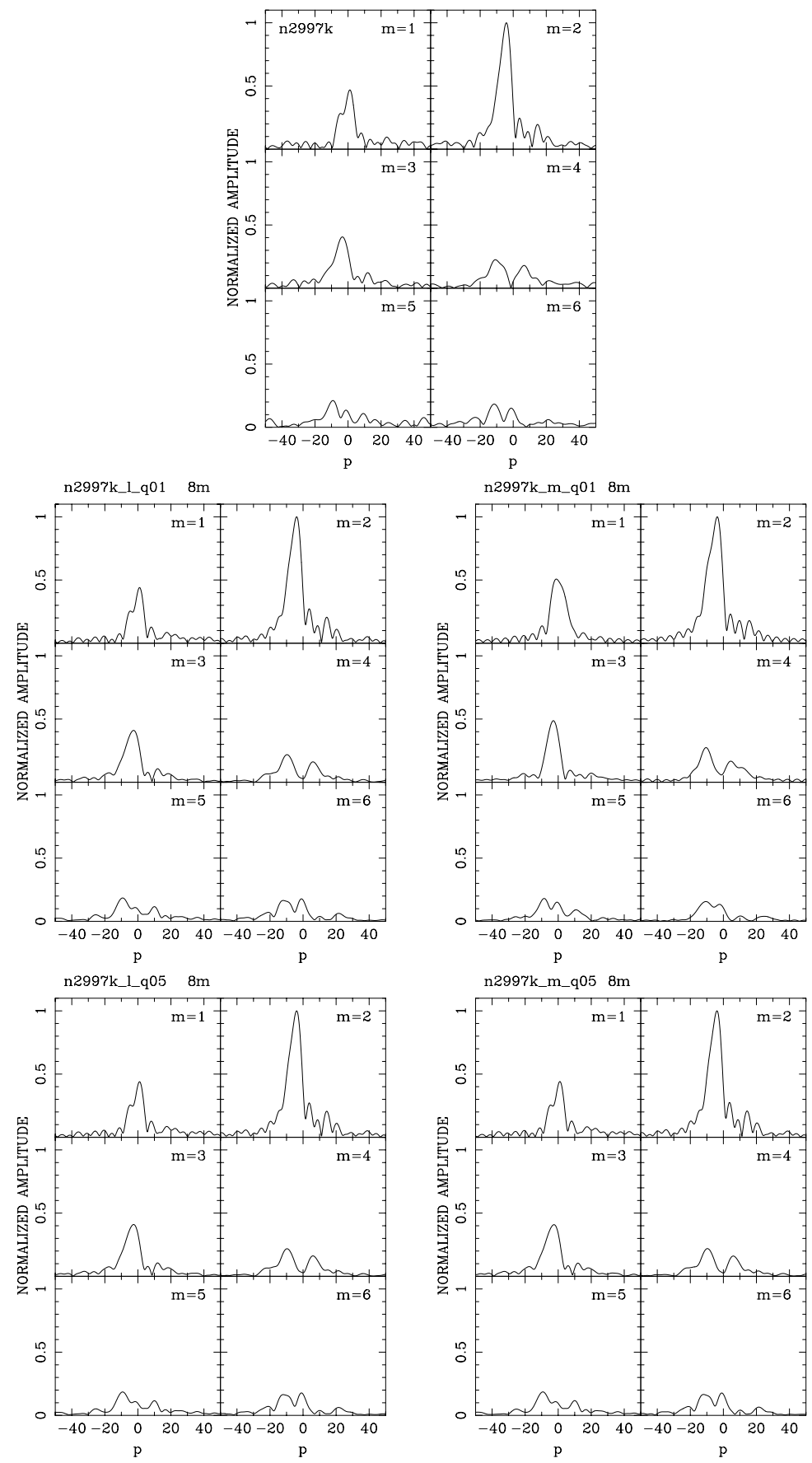

Fig. 9. As in Fig. 8. Illustrated in the uppermost two panels are restframe $K^{\prime}$ Fourier spectra of the grand design Sc spiral galaxy NGC 2997. The middle row shows the Fourier spectra when NGC 2997 is moved to redshifts $z=0.7$ ( $L$ band) and $z=1.2$ ( $M$ band), respectively. We adopt a deceleration parameter of $q_{0}=0.1$. The bottom row shows the Fourier spectra for the same redshift values, but assuming a different cosmology with $q_{0}=0.5$

strong in amplitude as the $m=1$ component (Fig. 8), and corresponds to the bisymmetric spiral seen at $K^{\prime}$ which lies within the arc (Fig. 1). What is intriguing about the $m=2$ component in Fig. 8 is that it is doubly peaked at opposite sides of the origin of the $p$-axis, indicative of a set of leading and trailing wave modes which are propagating inward and outward, being reflected off a central bulge (see Puerari et al. 2000). In other words, NGC 922 shows the unmistakable signature of spiral arm modulation in its stellar disk. Spiral arm modulation has hitherto only been detected in grand design disks (such as Messier 81). With these beating mode considerations, NGC 922 could well be a grand design spiral in the process of assembly. In our template of Figs. 5 and. 7, the bisymmetric spiral in NGC 922 would carry the classification $\mathrm{H} 2 \gamma$.

NGC 922 has a gravitational bar strength or torque (Buta \& Block 2001) of 2 (on a scale of 0 to 6). 


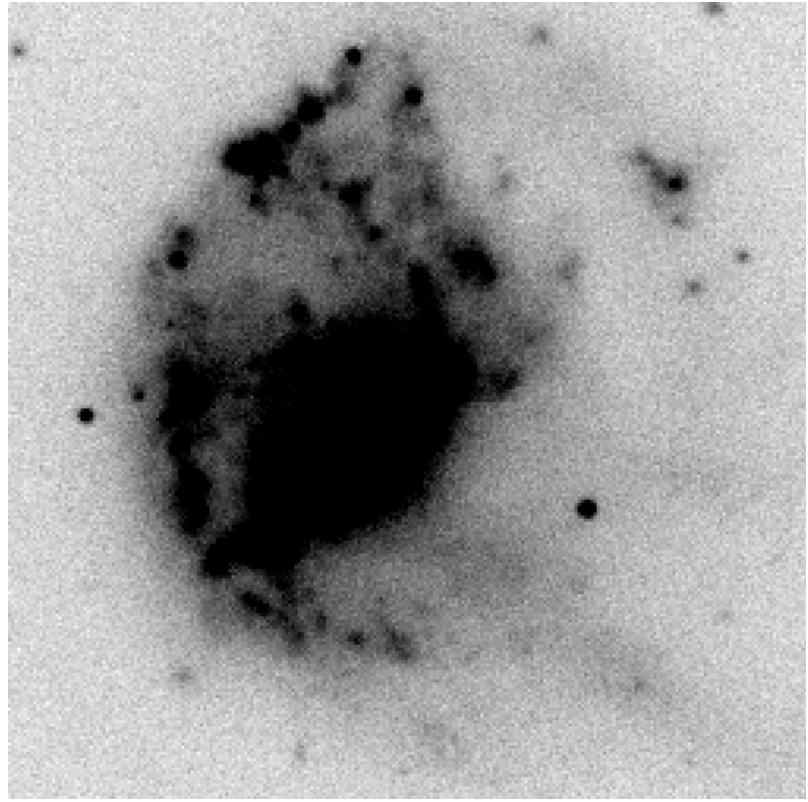

Fig. 10. NGC 922 could well serve as a local Rosetta stone for morphologically peculiar systems in our higher redshift Universe. Apparent chaos reigns supreme in this deep optical $B$-band image of NGC 922; dust-penetrated imaging, however, shows that a simple two-armed spiral (betraying the signature of arm modulation found in several grand design prototypes such as Messier 81) is largely responsible for the dynamics in the stellar backbone of NGC 922 whose Fourier spectra are presented in Fig. 8

The complete quantitative classification of NGC 922 (focussing on the inner spiral seen at $K^{\prime}$ ) is therefore $\mathrm{H} 2 \gamma 2$.

The bar strength component of this classification scheme is particularly interesting, given the evidence for a rapid decline in the proportion of optically barred galaxies at high redshifts (Abraham et al. 1999; van den Bergh et al. 2000). The corresponding behaviour in the restframe infrared, where bars are much more prominent in local data (Eskridge et al. 2000), is unknown.

It must be stressed that Fourier spectra of NGC 922 are being generated on square "postage stamp" FITS images only $3^{\prime \prime}$ on a side $(64 \times 64$ pixels, where 1 pixel corresponds to 50 mas), yet the appearance of the modes are strikingly similar to those of the local $z \sim 0$ spectra. The decrease of spatial resolution in higher redshift space does not wash out the dominant modes of NGC 922 seen locally (Fig. 8). In fact, given two sets of Fourier spectra, one for the galaxy at its original distance, and another, generated from the simulated image at $z=0.7$ or $z=1.2$, it is a challenge to find any noticeable difference in the dominant modes at all (independent of $q_{0}$ ). Identical conclusions pertain to the preservation of pitch angle in NGC 2997 (see Fig. 9). The class $\beta$ bin is retained throughout.

An interesting point to note is that the first and second order harmonics in Figs. 8 and 9 are not mutually exclusive. A galaxy often presents both $\mathrm{H} 1$ and $\mathrm{H} 2$ components. Modal theories of spiral structure predict that $m=1$ modes should generally be accompanied by $m=2$ modes when available, since the latter are more efficient in transporting angular momentum outwards. For example, the inner leading arm in NGC 4622 (labeled in Fig. 6a of Block et al. 1994b) co-exists with an outer set of $m=2$ spirals. Block et al. (2000) classify the lopsided arm in NGC 4622 as H1 $\alpha$ and the outer set of tightly wrapped spirals as $\mathrm{H} 2 \alpha$. What Figs. 8 and 9 do show is part of a general trend already noted by Block et al. (1994b) that modes higher than 2 (i.e. multi-arm features) are generally suppressed in stellar disks imaged at restframe $K^{\prime}$, whereas the dusty, gaseous components seen in optical images are dynamically very active, fueling Jeans instability and readily supporting multi-arm features.

A note on deprojections at high- $z$ is in order. By the time NGST is launched, IFU-based spectroscopy ought to be the norm (in fact, some form of resolved spectroscopic capability is likely to be a component of the NGST instrument load). Kinematical information can then be used, as in our local Universe, to secure accurate deprojection parameters. However, we do wish to point out that lessons from $z \sim 0$ galaxies show that deprojections based on axial ratios alone are excellent (kinematical studies simply finely-tune them). In our high- $z$ universe, axial ratios from even relatively low signal-noise NGST images could readily be determined from image moments; being based on calculations summed over many - rather than a few - pixels, they should be remarkably robust (see Frei 2000), even without spectroscopic/kinematical NGST information.

When high redshift galaxies are observed at the $L$ and $M$ bands with NGST, the expectations are that many will be spirals undergoing intense bursts of star formation (Ferguson \& Babul 1998; Takamiya 1999). To classify two-armed spirals such as NGC 2997 (Fig. 4) according to the templates in Figs. 5 and 7 is straightforward. According to the robustly determined Fourier spectra, its dust penetrated classification would be $\mathrm{H} 2 \beta$. The point of this investigation is that even if a large percentage of these high $z$ systems are optically as irregular as NGC 922, only two low order modes $(m=1$ and $m=2$ ) are invariably required to describe the dust penetrated disk, in agreement with the ubiquity of low-order stellar modes found in our local Universe (Block \& Wainscoat 1991; Block et al. 1994b, 2000).

\section{Conclusion}

The utility of an optical imaging capability for NGST is currently being debated (a list of potential instruments is listed on the NASA Webpage www701.gsfc.nasa.gov/isim/science.htm). The performance of such an instrument can, to a limited degree, be extrapolated from the current capability of HST. Owing to the paucity of work in this area, it has been less clear what one hopes to learn from morphological studies undertaken in the $L$ and $M$ near-infrared bands that are the focus of much of the NGST science case. 


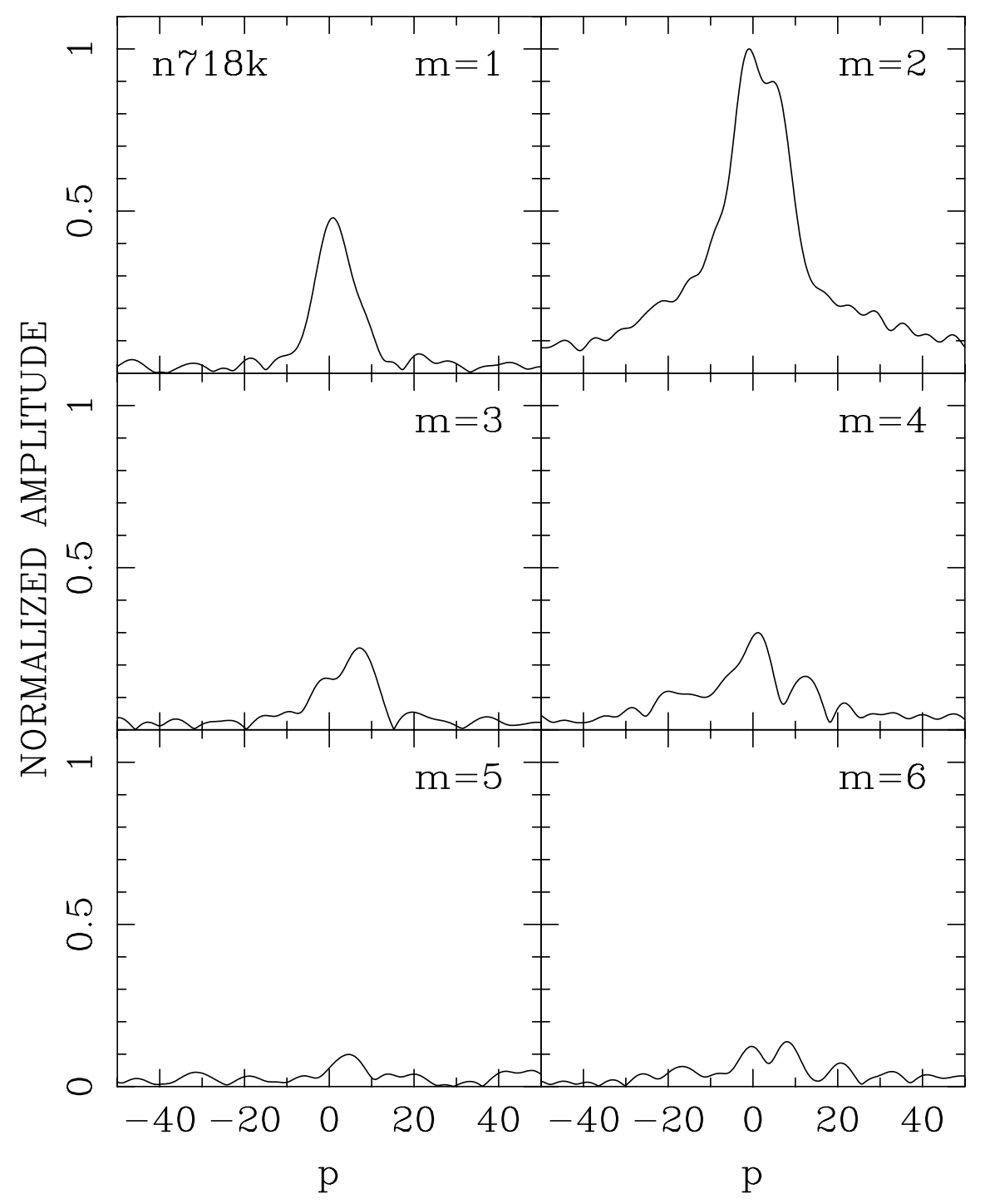

Fig. 11. Lessons from our local Universe show that the dust penetrated Fourier spectra of galaxies on opposite sides on the Hubble tuning fork may be almost identical. Seen here are the Fourier spectra generated from a $K^{\prime}$ image of NGC 718 (Hubble type a). Compare these spectra to those of NGC 309 (Hubble type c) in Fig. 12

Our goal in this paper has been to argue (from a single illustrative example) that important morphological work will be achieved at these longer wavelengths. We have identified NGC 922 as a reasonably representative "archetypal" candidate similar in appearance to high redshift morphologically peculiar counterparts (see Fig. 10). By simulating dust-penetrated images of this object at $z \sim 1$, and undertaking a detailed analysis of this synthetic data, we have shown that quantitative morphological investigation of such systems is well within the capabilities of the current NGST design.

Perhaps more importantly, we have argued for the necessity of such observations in order to interpret the nature of optically peculiar systems at high redshifts. Optical observations of the high redshift Universe may not effectively trace underlying baryonic mass distributions or "galactic backbones", but rather focus for example, on the degree of symmetry/asymmetry of the dusty Population I component. This is especially true in systems undergoing large bursts of star formation, where the focus in the optical or UV is on OB associations and HII regions. A crucial lesson from our local Universe is that galaxies on opposite ends of the Hubble tuning fork may present almost identical mass distributions, and belong to the same dustpenetrated class. This result is clearly seen in Figs. 11 and 12. It is not, a priori, possible to predict what galactic backbones might look like when the disks are dust penetrated at low, and at high, redshift. At redshifts greater than 1.5, substantial mass could be locked into "old" (ages greater than one or two Gyr) stellar populations at surface brightness levels that are too faint to be visible on deep HST images (Abraham 1998). Near-IR imaging with NGST may well provide the most effective means for probing both evolved and dust-obscured stellar components in such systems.

Acknowledgements. It is a pleasure to thank the referee, Dr. A. Moorwood, as well as Garth Illingworth and Stephane Charlot for some very helpful comments. AS is a Visiting 


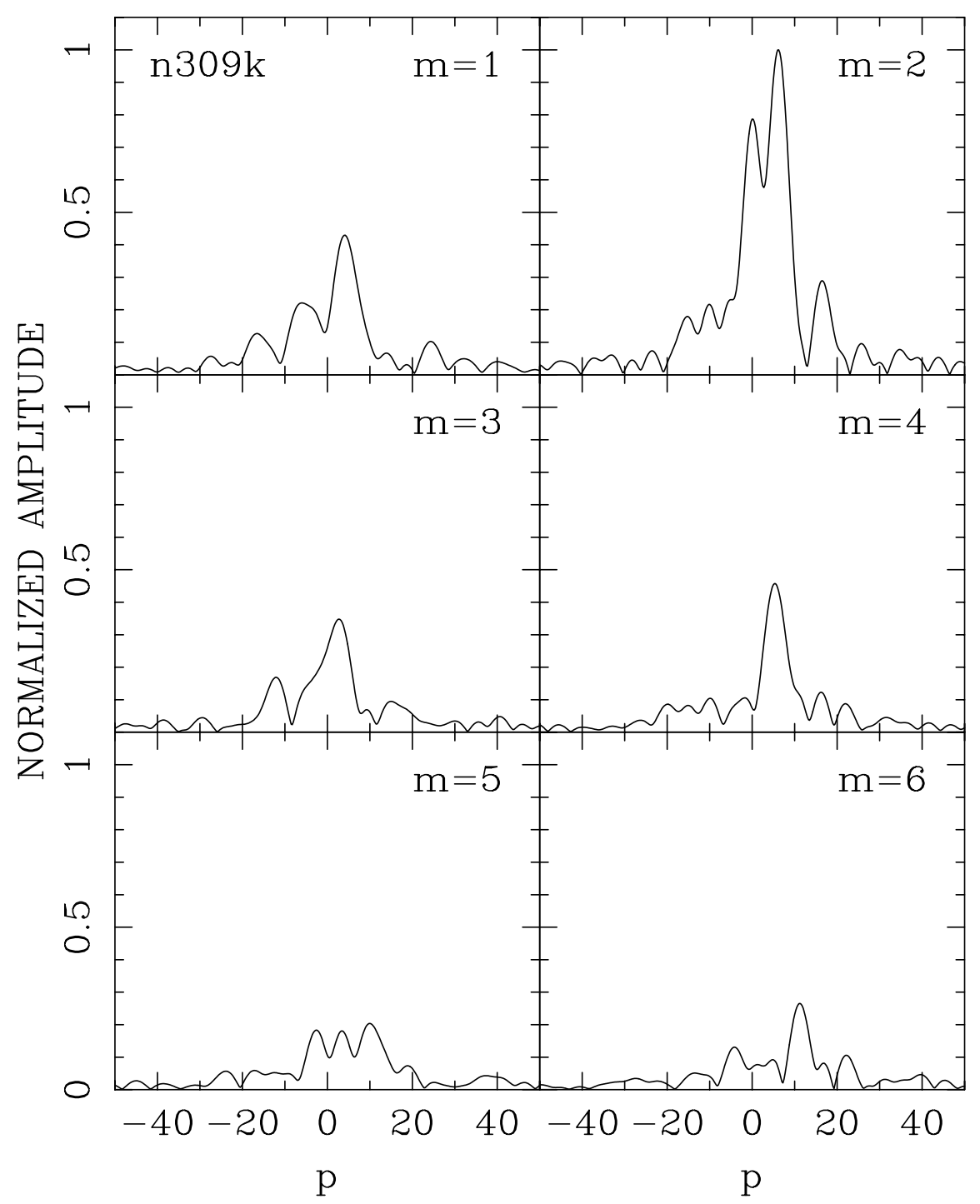

Fig. 12. The Fourier spectra generated from a type c galaxy (NGC 309) at restframe $K^{\prime}$ are remarkably similar to those presented in Fig. 11 of NGC 718 (type a). Both NGC 309 and NGC 718 belong to the same dust penetrated bin $\beta$, and nearinfrared images of these galaxies may be found in Fig. 7. The galactic backbones of NGC 718 and NGC 309 are very similar, despite the fact that optically, these two galaxies are located on opposite ends of the Hubble tuning fork

Astronomer at the Infrared Telescope Facility, which is operated by the University of Hawaii under contract from the National Aeronautics and Space Administration. DLB, IP and AS are indebted to the Anglo-American Chairman's Fund Educational Trust. A note of deep appreciation is expressed to Mrs. M. Keeton and the Board of Trustees. Dr. Rolf Chini is also warmly thanked for his input. This research is partially supported by the Mexican Foundation CONACYT under the grant No. 28507-E.

\section{References}

Abraham, R. G., et al. 1996a, MNRAS, 279, L47

Abraham, R. G., van den Bergh, S., Glazebrook, K., et al. 1996b, ApJS, 107, 1

Abraham, R. G. 1998, Perspectives in Physical Morphology, Les Houches Summer School [astro-ph/9809131]

Abraham, R. G., et al. 1999, MNRAS, 308, 569

Bally, J., \& Morse, J. A. 1999, Amer. Astron. Soc. Meet., 195, 86
Block, D. L., \& Wainscoat, R. J. 1991, Nature, 353, 48

Block, D. L., Witt., A. N., Grosbøl, P., Stockton, A., \& Moneti, A. 1994a, A\&A, 288, 383

Block, D. L., Bertin, G., Stockton, A., et al. 1994b, A\&A, 288, 365

Block, D. L., \& Puerari, I. 1999, A\&A, 342, 627

Block, D. L., Puerari, I., Frogel, J. A., et al. 2000, in Toward a New Millennium in Galaxy Morphology, ed. D. L. Block, I. Puerari, A. Stockton, \& dW. Ferreira (Kluwer Dordrecht), 5

Bouwens, R., Broadhurst, T., \& Silk, J. 1998, ApJ, 506, 557

Buta, R. J., \& Block, D. L. 2001, ApJ [astro-ph/0010342]

Charlot, S. 1996, in The Universe at High Redshift, ed. E. Martinez-Gonzalez, \& J. L. Sanz (Springer: Lecture Notes in Physics), 470, 53

Charlot, S. 1998, in NGST - Science Drivers \& Technological Challenges, 34th Liege Astrophysics Colloquium, ed. P. Benvenuti, et al., ESA-SP 429, 135 [astro-ph/9810408]

Cohen, J. G. 1976, AJ, 203, 587

Danver, C. G. 1942, Lund. Obs. Ann., vol. 10 
Devereux, N. A. 1989, ApJ, 346, 126

Driver, S. P., et al. 1998, ApJ, 496, L93

Eskridge, P. B., Frogel, J. A., Pogge, R. W., et al. 2000, AJ, 119,536

Ferguson, H. C., \& Babul, A. 1998, MNRAS, 296, 585

Frei, Z. 2000, in Toward a New Millennium in Galaxy Morphology, ed. D. L. Block, I. Puerari, A. Stockton, \& dW. Ferreira (Kluwer Dordrecht), 577

Frogel, J. A., Quillen, A. C., \& Pogge, R. W. 1996, in New Extragalactic Perspectives in the New South Africa, ed. D. L. Block, \& J. M. Greenberg (Kluwer Dordrecht), 65

Giavalisco, M., Livio, M., Bohlin, R. C., Machetto, F. D., \& Stecher, T. P. 1996, ApJ, 112, 369

Glazebrook, K. et al. 1995, MNRAS, 275, 157

Gillett, F., \& Mountain, M. 1998, ASP Conf. Ser., 133, 42

Hauser, M. G. 1994, Amer. Astron. Soc. Meet., 185, 32

Holland, W., Robson, E., Gear, W., et al. 1999, MNRAS, 303, 659

Hughes, D. H. 1996, in Cold Gas at High Redshift, ed. M. N. Bremer, P. P. van der Werf, H. J. A. Röttgering, \& C. L. Carilli (Kluwer Dordrecht), 321
Jenness, T., \& Lightfoot, J. F. 1998, ASP Conf. Ser., 145, 216 Kalnajs, A. J. 1975, in La Dynamique des Galaxies Spirales, ed. L. Weliachew (Paris Éditions du CNRS), 103

Kauffmann, G., \& Charlot, S. 1998, MNRAS, 297, L23

Puerari, I., Block, D. L., Elmegreen, B. G., Frogel, J. A., \& Eskridge, P. B. 2000, A\&A, 359, 932

Puerari, I., \& Dottori, H. A. 1992, A\&AS, 93, 469

Sandage, A., \& Bedke, J. 1994, The Carnegie Atlas of Galaxies, Carnegie Institution of Washington Publication, No. 638

Sanders, D. B. 2000, in Toward a New Millennium in Galaxy Morphology, ed. D. L. Block, I. Puerari, A. Stockton, \& dW. Ferreira (Kluwer Dordrecht), 381

Schröder, M. F. S., Pastoriza, M. G., Kepler, S. O., \& Puerari, I. 1994, A\&AS, 108, 41

Takamiya, M. 1999, ApJS, 122, 109

van den Bergh, S., Abraham, R. G., Ellis, R. S., et al. 1996, AJ, 112, 359

van den Bergh, S. 1998, Galaxy Morphology and Classification (Cambridge University Press Cambridge), 87

van den Bergh, S., et al. 2000, AJ, 120, 2190 